\title{
Multi-Chip RFID Antenna Integrating Shape-Memory Alloys for Detection of Thermal Thresholds
}

\author{
Stefano Caizzone, Cecilia Occhiuzzi, and Gaetano Marrocco
}

\begin{abstract}
Low-cost wireless measurement of objects' temperature is one of the greatest expectation of radiofrequency identification technology for the so many applications in cold supplychain control and safety assessment in general. In this context, the paper proposes a dual-chip UHF tag embedding shape memory alloys (SMA) able to transform the variation of the tagged item's temperature into a permanent change of antenna radiation features. This event-driven antenna is hence able to selectively activate the embedded microchips according to the temperature above or below a given threshold. A general design methodology for the resulting two-ports tag antenna is here introduced and then applied to prototypes able to work at low (around $0^{\circ} \mathrm{C}$ ) and high $\left(80^{\circ} \mathrm{C}\right)$ temperatures.
\end{abstract}

Index Terms-Radiofrequency Identification (RFID), shape memory alloy, temperature sensor.

\section{INTRODUCTION}

$\mathbf{P}$ ASSIVE UHF tags for radiofrequency identification (RFID) are often considered as digital devices which, when interrogated and energized by a reader, may send back their own ID or other resident information through a backscattering modulation of the incident continuous wave [1]. While the most assessed use is the item labeling, there is a growing interest in new applications devoted to sense the variation of the tagged objects as well as of the nearby environment [2]-[6].

One of the most interesting parameters to be sensed is the temperature variation for a large variety of scopes, in logistic, safety and medical contexts. In particular, cold supply chain management for goods that have to be kept at constant temperature has a severe need for electronic tags able to continuously detect possible thermal anomalies [7], [8]. Nowadays, the most common devices for temperature monitoring are active data loggers [9] which, by means of active sensors and local data storage, are able to store all the time-history of the temperature. The relative high costs and the limited lifetime before recharge make these systems only useful for an environmental-level monitoring, leaving unsolved the problems related to the pervasive item-level control.

An alternative and cheaper approach is represented by semiactive RFID temperature tags [10], having a longer lifetime and

Manuscript received June 21, 2010; revised October 26, 2010; accepted November 19, 2010. Date of publication May 10, 2011; date of current version July 07,2011 . This work was supported by the Italian Ministry of University under project PRIN-2008- RFID MULTITAG.

S. Caizzone is with the Universita di Roma Tor Vergata, DISP, 00133 Roma, Italy and also with the Antenna Group, Institute of Communications and Navigation, German Space Agency (DLR), 82230 Wessling, Germany.

C. Occhiuzzi and G. Marrocco are with the Universita di Roma Tor Vergata, DISP, 00133 Roma, Italy (e-mail: marrocco@ disp.uniroma2.it).

Digital Object Identifier 10.1109/TAP.2011.2152341 able to transmit the instantaneous value of the local temperature following the reader's interrogation. Their main drawback is that the thermal event can be detected only in the presence of a close-distance reader and hence they are not suited to assess the thermal safety all along the supply chain.

A possibility to reduce cost, size and maintenance of RFID sensor systems is to focus the attention to a completely passive technology where the challenge is to design a proper passive sensor component able to perform the sensing activity without batteries and to store the occurrence of the temperature hot spot independently on the reader interrogation. Following this idea, a UHF passive sensing tag which integrates a temperature printed nano sensor has been recently proposed in [11]. The sensor works as a chemical thermal fuse able to record the thermal event by irreversibly changing its resistivity through the melting of the polymeric part of the conducting ink. However, it currently looks suitable only for high temperature applications, approximately over $60^{\circ}$, and not for typical cold supply chain conditions.

In [6] a similar idea was instead applied to low temperature, wherein the switch effect was obtained by the melting of an iced region placed in the close proximity of the antenna and hence the device is restricted to applications around $0^{\circ} \mathrm{C}$ without many tuning possibility.

This paper proposes a completely different mechanism to achieve one-shot RFID thermal seals, fully suitable to high and low temperatures, in a range approximately between $-30^{\circ} \mathrm{C}$ and $100^{\circ} \mathrm{C}$. A shape-memory-alloy (SMA) compound, introduced into an RFID tag as sensitive material, modifies its geometry depending on the local thermal boundary conditions and consequently it is able to modulate the RFID communication.

The sensing tag is here conceived as an antenna provided with two RFID microchips so that the first one (chip-1) is able to transmit its digital identifier (ID1) regardless the temperature status, while the second chip (chip-2) transmits or not its code (ID2) depending on the local temperature. Hence ID1 has the meaning of item's identifier while ID2 gives its status information, e.g. a thermal alarm (above or below a threshold temperature), according to the ID-modulation paradigm [12]. For this purpose, a portion of the antenna (Fig. 1) is fabricated in SMA whose shape variation with respect to the temperature will be such to match or mismatch the chip-2, leaving mostly unmodified the impedance condition seen by chip-1. The occurrence of a hot spot is hence registered into the device by a shape change, independently on the presence of the reader.

The design of such a new family of devices deserves some care for what concerns the management of multi-chip RFID tags, and a proper methodology is here proposed able to take into account the two-state response of the tag. 

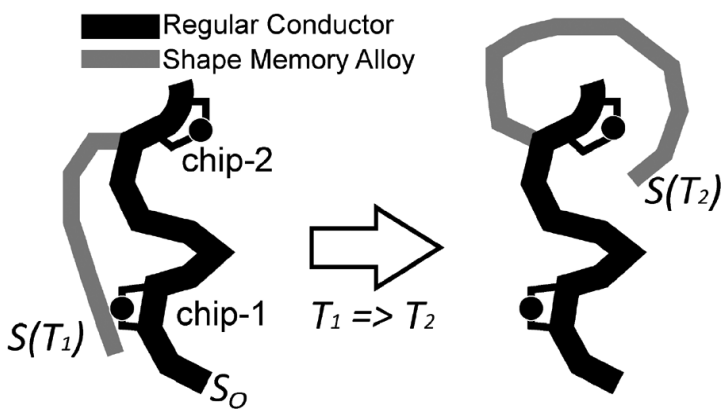

Fig. 1. Functional scheme of a two-chip temperature sensing tag embedding regular conductors of shape $S_{0}$ and Shape Memory compounds whose shape $S$ is strongly affected by the local temperature.

The paper is organized as follows: Section II introduces a possible configuration of thermal switch, suited to antenna integration and discusses, by means of some experiments, the repeatability of its thermal response. Section III formulates the design problem of the two-port RFID tags including the event-driven sensor's status. A tag prototype is then designed and electromagnetically characterized in Section IV and its response is finally experimented in Section $\mathrm{V}$ at hot (around $80^{\circ} \mathrm{C}$ ) and cold (around $0^{\circ} \mathrm{C}$ ) temperature conditions.

\section{The Nitinol Thermal Switch}

Shape memory alloys [13] are characterized by two crystallographic phases, a martensitic one and an austenitic one, in which the structural and mechanical properties of the alloy greatly change. By heating, martensite transforms into austenite, the only stable phase at high temperature. SMAs are easily deformable in the martensitic phase; however they recover the prefixed austenitic shape every time they are heated above the transition $A_{S}$ temperature (Austenite Start), with an increasing recovery as the temperature rises up to $A_{F}$ (Austenite Finish). According to their property to recover the "memorized" shape, the SMAs can be employed both as sensors and/or as actuators.

The shape-memory effect has been found in many alloys such as $\mathrm{CuAlNi}$, CuZnAl, AuCd, but NiTi, also known as Nitinol [14], is the most commonly used thanks to its good characteristics of corrosion resistance, ductility, high recoverable deformation, relatively high electrical conductivity $\left(\sigma_{N i T i}=1.210^{7} \mathrm{~S} / \mathrm{m},[15]\right)$ and biocompatibility. Nitinol is nowadays widely used in medicine and robotics mainly to build actuators. In the Electromagnetic context Nitinol wires are instead used to fabricate flexible whip antennas for mobile terminals.

It is worth mentioning that the transition temperature $A s$ is tunable by changing the alloy percentage composition: therefore transitions can be obtained in a very wide range of temperatures, i.e. between $-30^{\circ} \mathrm{C}$ and more than $100^{\circ} \mathrm{C}$. This unique feature makes the Nitinol an enabling compound to assess the integrity of pharma, blood sacs, frozen foods and to monitor fever rush or industrial processes in general.

\section{A. Switch Geometry}

There are several options to achieve a temperature-sensitive tag taking advantage of the shape recovering feature of the Nitinol, but one of the easiest is to conceive a thermal switch which is normally closed when the temperature $T<A_{S}$ and
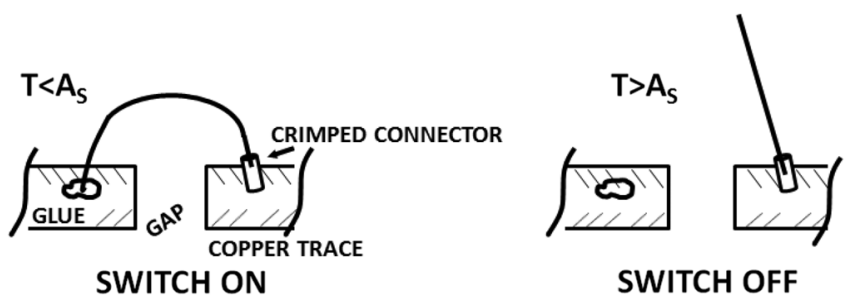

Fig. 2. Geometry of the Nitinol temperature switch in the martensite and austenite states.

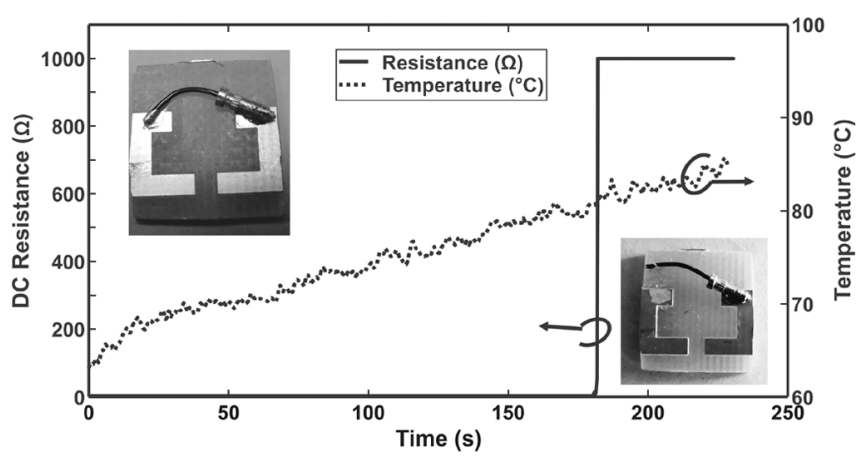

Fig. 3. Example of fabricated Nitinol switch and graphical representation of the switch-off with respect to the temperature rising.

open for $T>A_{S}$. The switch can be placed in series or in parallel to the chip-2 (see again Fig. 1) to strongly affect its activity. According to the last case, the tag' states will be: ID1 under thermal threshold, and ID1+ID2 over the thermal threshold. A possible implementation of a thermal SMA-based switch, suitable to RFID antenna integration, includes a Nitinol wire segment processed to have straight shape in the austenitic (stable) phase. The wire is bent (Fig. 2) in the martensitic phase, e.g., for $T<A_{S}$, and fixed across the gap of a copper trace (wherein the RFID microchip will be placed) by a crimped connector ${ }^{1}$ soldered at one of the gap's sides, and more softly, by a silver glue, at the other side. When the temperature exceeds the threshold $\left(T>A_{S}\right)$, the wire begins to recover its original straight shape, breaking the conducting glue and hence opening the switch.

Since the austenitic shape is stable, a successive fall of the temperature below the threshold will not modify the wire's shape anymore. The Nitinol switch hence acts as a one-way element, configuring itself as an unidirectional thermal fuse.

\section{B. Prototype and Characterization}

Fig. 3 shows a fabricated example of a Nitinol switch over a FR4 substrate (thickness $1.58 \mathrm{~mm}$ ). Two Nitinol wires having a nominal transition temperature $A_{S}=80^{\circ} \mathrm{C}$ and different diameters $\{0.3 \mathrm{~mm}, 0.5 \mathrm{~mm}\}$, respectively, have been considered in separate devices. The same figure gives a graphical rendering of the switch-off capability of the device, when the temperature is linearly raised from $60^{\circ} \mathrm{C}$ to $85^{\circ} \mathrm{C}$ in a thermostatic chamber. The switch-off event has been collected by monitoring the DC resistance at the switch's terminals. A sharp variation of input DC resistance from low (switch on) to an extremely high value (switch off) indicates the transition from martensite to austenite,

\footnotetext{
${ }^{1}$ The Nitinol can not be easily soldered and hence a possible fixing is achieved by crimping the Nitinol wire within a conducting small cylinder and soldering this one on the antenna trace.
} 
TABLE I

SWITCH-OFF TEMPERATURE DETECTED BY THE CONSIDERED NITINOL SWITCHES

\begin{tabular}{c|c|c}
\hline Wire diameter $(\mathrm{mm})$ & $T_{S W}\left({ }^{\circ} \mathrm{C}\right)$ & $\sigma_{T}\left({ }^{\circ} \mathrm{C}\right)$ \\
\hline 0.3 & 81.7 & $1.44(1.7 \%)$ \\
\hline 0.5 & 75.3 & $2.7(3.1 \%)$ \\
\hline
\end{tabular}

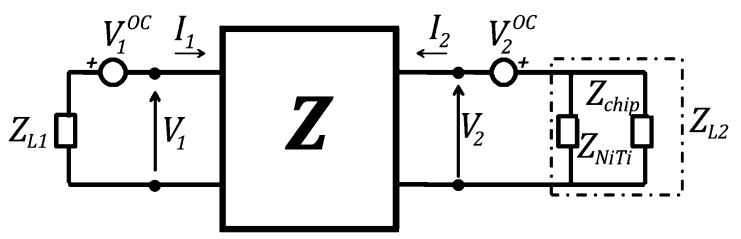

Fig. 4. Two-port model of the two-chip RFID tag in receiving mode.

and hence the required open circuit. The experiments have been repeated five times for each kind of switch and the detected transition temperatures in Table I demonstrate that the device mechanism has good repeatability, with a temperature uncertainty of $1-2^{\circ} \mathrm{C}$. The switch-off $\left(T_{S W}\right)$ temperature is dependent on the diameter of the wire so that a thicker Nitinol wire will recover the stable state at a lower temperature in comparison with a thinner wire and hence this one is more sensible.

From an electrical point of view, the Nitinol wire can be simply modeled by a lumped impedance $Z_{N i T i}^{O N / O F F}$, and in particular as an ideal open circuit when in the austenite phase $\left(Z_{N i T i}^{O F F}=\infty\right)$, and as a real inductor $\left(Z_{N i T i}^{O N}=R_{N i T i}+\right.$ $\left.j \omega L_{N i T i}\right)$ with parameters

$$
\begin{aligned}
R_{N i T i} & =\rho \frac{l}{A} \\
L_{N i T i} & =2 l\left[\ln \left(\frac{2 l}{r_{0}}\right)-1\right]
\end{aligned}
$$

in the martensite phase, where $l$ and $r_{0}$ are the length and the radius of the Nitinol wire segment, respectively [16].

\section{TWO-CHIP TAG ANTENNA DESIGN}

The passive RFID communication between reader and tags requires the input impedance of the antenna to be matched to the conjugate of microchip impedance for maximum power transfer (and hence for maximum reading range) [1]. Since the two RFID microchips are connected over a same radiating structure, the electromagnetic coupling can not be neglected and hence the input impedances to be controlled are the active impedances of the system [17]. The design problem can be conveniently formulated in terms of a two-port antenna system in receiving mode. The corresponding network model is given in Fig. 4: $Z_{L 1}=Z_{\text {chip }}$ is the load impedance at port-1, wherein the chip-1 is connected, while $Z_{L 2}=Z_{\text {chip }} \| Z_{N i T i}$ is the terminating impedance of port-2, which depends on the ON/OFF status of the Nitinol switch, and hence on the sensed temperature. $V_{1,2}^{O C}$ are the open circuit voltages collected at the ports and produced by the interrogating field emitted by the remote reader. $\mathbf{Z}$ is the impedance matrix of the unloaded two-port antenna.

The network equations [18] are

$$
\begin{aligned}
& Z_{11} I_{1}+Z_{12} I_{2}=V_{1}=-V_{1}^{O C}-Z_{L 1} I_{1} \\
& Z_{21} I_{1}+Z_{22} I_{2}=V_{2}=-V_{2}^{O C}-Z_{L 2} I_{2} .
\end{aligned}
$$

The open circuit voltages depend on the geometry of the tag but also on the mutual orientation between reader and tag. Therefore, as in the case of a regular array, the active impedances of the tag are strongly affected by the interrogation modality. The general formulation of this kind of multi-chips device, also denoted as "RFID grids," is out of the scope of this paper and can be found in [19]. Under broadside incidence condition (e.g., the plane of the tag is perpendicular to the reader's antenna beam), it is possible to assume $V_{1}^{O C}=V_{2}^{O C}$ and the above system equation gives

$$
\frac{I_{2}}{I_{1}}=\frac{Z_{11}-Z_{21}+Z_{L 1}}{Z_{22}-Z_{12}+Z_{L 2}} .
$$

Accordingly, the active impedances for broadside interrogation are

$$
\begin{aligned}
& Z_{i n, 1}=Z_{11}+\alpha Z_{12} \\
& Z_{i n, 2}=Z_{22}+\frac{Z_{12}}{\alpha}
\end{aligned}
$$

where $\alpha=\left(Z_{11}-Z_{21}+Z_{L 1}\right) /\left(Z_{22}-Z_{12}+Z_{L 2}\right)$. The maximum power transfer from the antenna to the microchip loads imposes [20] the following conjugate matching conditions at the ports:

$$
\begin{aligned}
Z_{i n, 1} & =Z_{c h i p}^{*} \\
Z_{i n, 2} \| Z_{N i T i} & =Z_{c h i p}^{*}
\end{aligned}
$$

By recalling the ID-modulation [12] scheme to be implemented in the sensing RFID tag, e.g. that only the chip- 1 is matched in the ON state $\left(T<A_{S}\right)$, while both the microchips are active in the OFF state $\left(T>A_{S}\right)$, the matching conditions to enforce on the two-ports tag are

$$
\begin{aligned}
& \text { switch } O N:\left\{\begin{array}{l}
\left|Z_{i n, 1}-Z_{\text {chip }}^{*}\right| \rightarrow 0 \\
\left|Z_{i n, 2}\right|\left|Z_{N i T i}^{O N}-Z_{\text {chip }}^{*}\right|^{-1} \rightarrow 0
\end{array}\right. \\
& \text { switch } O F F:\left\{\begin{array}{l}
\left|Z_{i n, 1}-Z_{\text {chip }}^{*}\right| \rightarrow 0 \\
Z_{\text {in, }, 2}\left|Z_{N i T i}^{O F F}-Z_{\text {chip }}^{*}\right| \rightarrow 0
\end{array}\right.
\end{aligned}
$$

The geometrical parameters of the two port antenna have to be therefore designed such to achieve the best trade-off among the above conditions. The following fitness function

$$
\begin{array}{r}
F=w_{11}\left|Z_{i n, 1}-Z_{c h i p}^{*}\right|+w_{12}\left|Z_{i n, 2}\right|\left|Z_{N i T i}^{O N}-Z_{c h i p}^{*}\right|^{-1} \\
+w_{22}\left|Z_{\text {in,2 }}-Z_{\text {chip }}^{*}\right|
\end{array}
$$

has thus to be minimized by means of a conventional optimization tool ( $w_{m n}$ are constant weights), having assumed $Z_{N i T i}^{O F F}=$ $\infty$ as discussed in the previous section.

\section{PRototype}

A first prototype of the UHF RFID thermal seal consists of a layout as in Fig. 5 originated from the single-port design in [21] where a planar dipole has been partly folded to reduce the overall area to a typical credit card size. The port impedances are controlled by the aspect ratio of two symmetric T-match circuits. The Nitinol switch has been fabricated as previously described by using a Nitinol wire of radius $r_{0}=0.5 \mathrm{~mm}$ and length $l=1 \mathrm{~cm}$. Accordingly, the closed-state impedance from (1) and (2) is $Z_{N i T i}^{O N}=0.1+j 37 \Omega$. The RFID microchips 


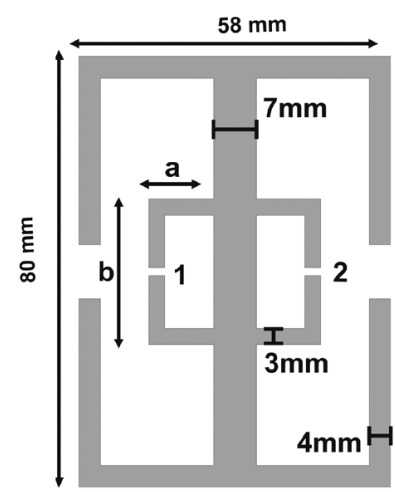

Fig. 5. Layout of the two-chip tag whose double T-match aspect ratio $(a, b)$ has to be optimized to achieve the required ID modulation.

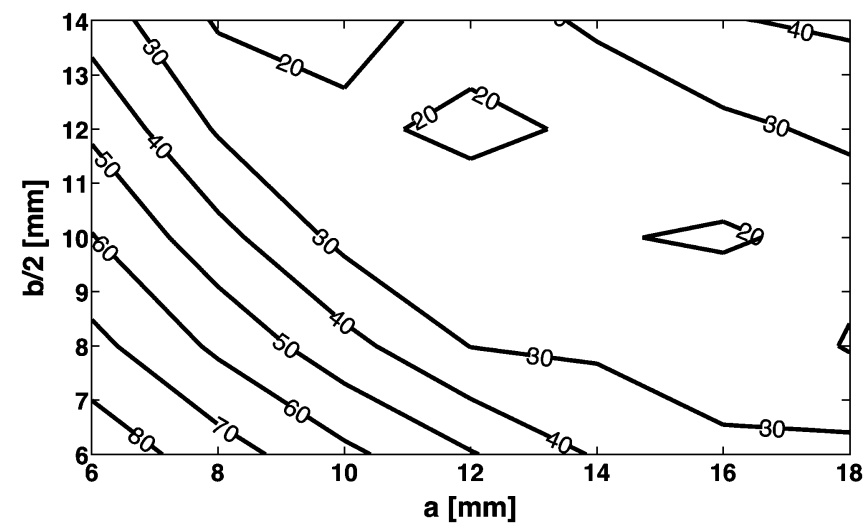

Fig. 6. Map of the fitness function $F(a, b)$ in equation (11) calculated by FDTD simulations.

are NXP TSS OP8 with nominal input impedance at $869 \mathrm{MHz}$ $Z_{\text {chip }}=15-j 135 \Omega$ and power sensitivity $P_{\text {chip }}=-15 \mathrm{dBm}$.

For the sake of simplicity, the T-match circuits are assumed to be of equal sizes $\{a, b\}$ whose values are selected by minimization of the fitness function $F(a, b)$ in (11) with respect to weights $w_{11}=0.85 w_{12}=0.05, w_{22}=0.1$. The antenna's $\mathbf{Z}$ matrix, for each shape of the T-match, is evaluated by an FDTD model [22] of the tag layout. Fig. 6 shows the map of $F(a, b)$ : a solution suited to fabrication is $a=12 \mathrm{~mm}$ and $b=25 \mathrm{~mm}$. The prototype has been cut on adhesive copper sheet as shown in Fig. 7 with a detail of the Nitinol wire insertion.

The electromagnetic tag performances in ON and OFF states have been experimentally characterized with respect to the realized gain which directly imposes the tag's read distance. For this purpose a customized UHF long-range reader based on the ThingMagic M5-e ASIC has been used. The sensing tag is interrogated by means of a $6 \mathrm{dBi}$ circular polarized patch antenna, connected to the reader. The tag is placed in front of the reader's antenna, at $1.5 \mathrm{~m}$ from ground and the floor reflections are minimized by using absorbing panels. Under the assumption of free-space channel, the power $P_{R \rightarrow T, n}$ collected by the $n$th port of the tag may be obtained using the Friis formula as

$$
P_{R \rightarrow T, n}=\left(\frac{\lambda_{0}}{4 \pi d}\right)^{2} G_{R}(\theta, \phi) \widetilde{G}_{T, n}(\theta, \phi) P_{i n}
$$

where $G_{R}$ is the gain of the reader antenna, $\widetilde{G}_{T, n}$ is the realized gain of the tag which includes the impedance mismatching between the $n$th port and the microchip as well as the polarization

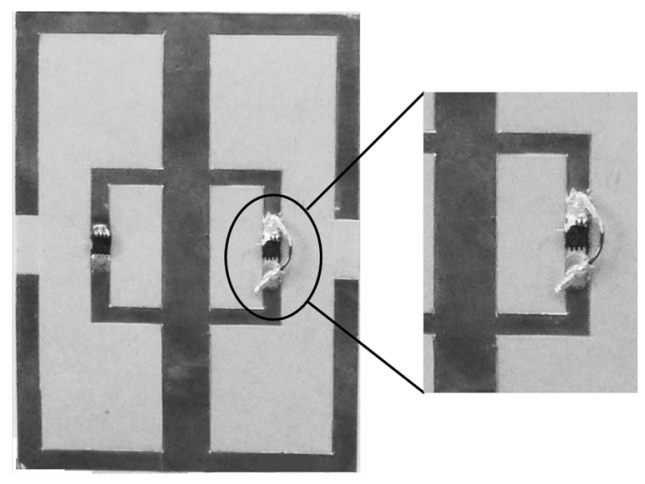

Fig. 7. Adhesive copper-sheet prototype of the tag embedding two microchips and the Nitinol switch, deduced from Fig. 5 with $a=12 \mathrm{~mm}$ and $b=25 \mathrm{~mm}$ The Nitinol wire length and diameter are $l=1 \mathrm{~cm}$ and $r_{0}=0.5 \mathrm{~mm}$, respectively.

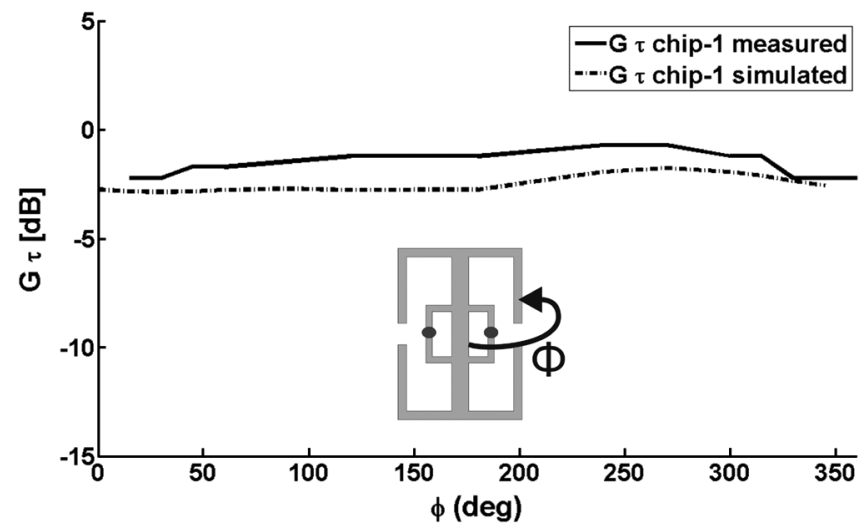

Fig. 8. Measured and simulated realized gain for the sensor-tag when the temperature is under the threshold $\left(T<A_{S}\right)$ and only chip-1 is responding.

mismatch with respect to the reader's field. $P_{i n}$ is the power accepted by the antenna of the reader unit. The realized gain is measured by means of the turn-on power method [23], e.g by recording the minimum power $P_{i n}^{t o}$ provided to the reader's antenna that enables the microchip on the tag to be activated and to send back its own identifier. In that condition $P_{R \rightarrow T, n}=P_{\text {chip }}$ and hence, for a fixed reader-tag distance $\left(d_{0}=0.8 \mathrm{~m}\right)$, the realized gain is found to be

$$
\widetilde{G}_{T, n}(\theta, \phi)=\frac{P_{\text {chip }}}{G_{R} P_{i n}^{t o}}\left(\frac{\lambda_{0}}{4 \pi d_{0}}\right)^{-2} .
$$

Fig. 8 and Fig. 9 give the measured $\widetilde{G}_{T, n}(\theta=\pi / 2, \phi)$, at $870 \mathrm{MHz}$, over the horizontal plane in both the Nitinol states. When the switch is closed and hence only the chip-1 is active, the realized gain over the H-plane is rather omnidirectional, with average value close to $-1 \mathrm{~dB}$. When the switch is open both the microchips are responding and the active radiation diagrams become less omnidirectional with specular gains of the two chips with respect to $\phi=180^{\circ}$. About $3 \mathrm{~dB}$ ripple and lower values are evident with respect to the single port case due to the presence of the inter-port coupling. Under the assumption of free space conditions and interrogation power $3.2 \mathrm{~W}$ EIRP, the measured realized gain achieves a maximum read distance (by inverting (13)) given by $d_{\max }\left(T<A_{S}\right)=8 \mathrm{~m}$ and $d_{\max }\left(T>A_{S}\right)=6 \mathrm{~m}$, below and above the threshold temperature, respectively. 


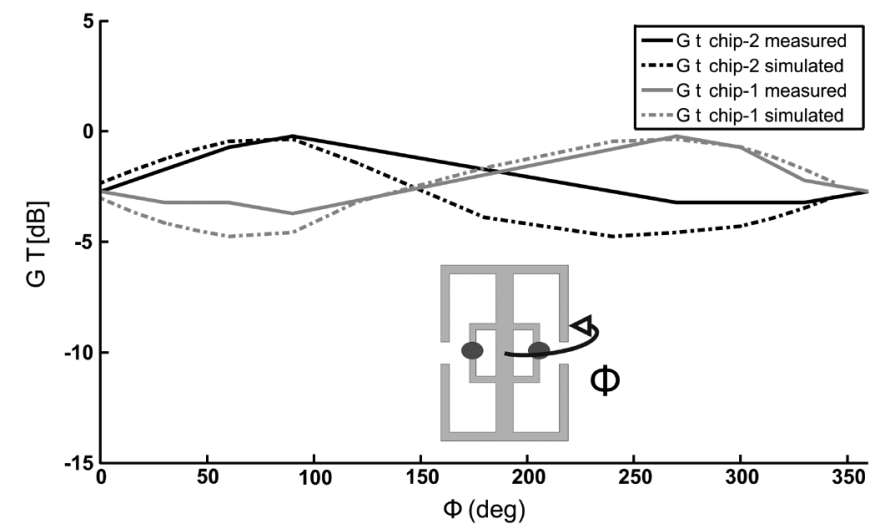

Fig. 9. Measured and simulated realized gain for the sensor-tag when the temperature is over the threshold $\left(T>A_{S}\right)$ and both chips are responding.

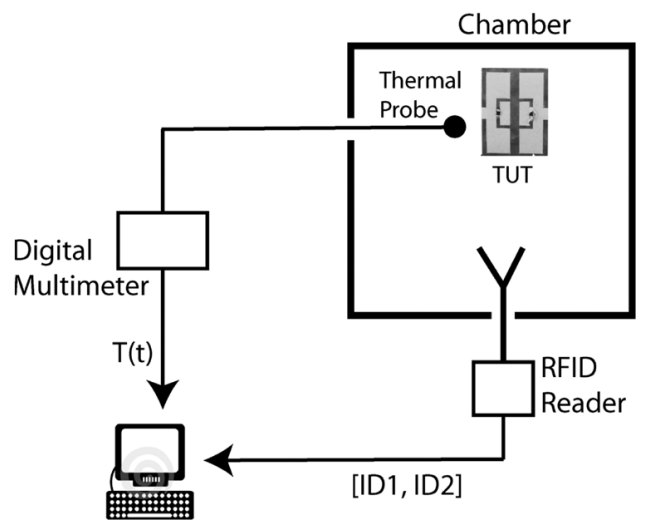

Fig. 10. Measurement setup comprising the remotely controlled reader, the multimeter connected to the thermocouple and the RFID tag under test (TUT), placed into the thermostatic chamber.

\section{THERMAL CHARACTERIZATION OF THE TAG}

The proposed RFID sensor has been tested in real conditions in order to verify the effective communication and sensing performances. Two different scenarios have been reproduced: a first one characterized by a high-temperature environment and a second one with a very low-temperature condition as for the logistics of frozen items. The Nitinol switch has been accordingly fabricated with wires of nominal transition temperature $A_{S} \simeq 80^{\circ} \mathrm{C}$ and $A_{S} \simeq-0^{\circ} \mathrm{C}$, respectively. The same antenna layout, shown in Fig. 7 has been used for both the experiments.

The tag has been attached on a cardboard box and heated inside a temperature controlled chamber, while being monitored by a thermal probe and continuously interrogated by the reader antennas also placed inside the chamber itself (Fig. 10). The tag's digital responses have been registered while the local temperature was progressively raised from a $T_{\text {init }}<A_{S}$ up to observe the martensite to austenite transition. The temperature was recorded through a thermocouple connected to a multimeter and correlated to the digital responses of the tag. The reader collects only the ID1 below the $A_{S}$ temperature. When both ID1 and ID2 are received for the first time it means that the Nitinol switch has broken the glue and the switch-off temperature $T_{S W}$ can be accordingly detected through the thermocouple reading.

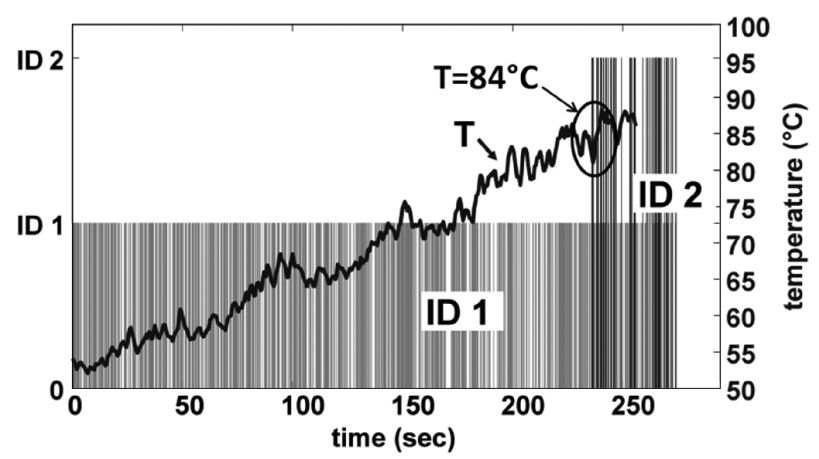

Fig. 11. Digital response from sensing tag with $A_{S} \simeq 80^{\circ} \mathrm{C}$ versus temperature raise and time.

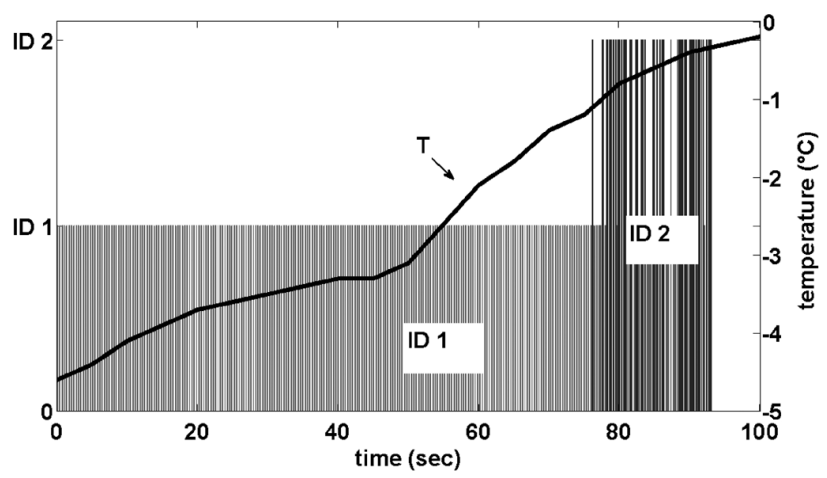

Fig. 12. Digital response from sensing tag with $A_{S} \simeq 0^{\circ} \mathrm{C}$ versus temperature increase and time.

TABLE II

Switch-OFF TEMPERATURES OF THE SENSING TAGS

\begin{tabular}{c|c|c}
\hline nominal $A_{S}^{\circ}(C)$ & $T_{S W}\left({ }^{\circ} C\right)$ & $\sigma_{T}\left({ }^{\circ} C\right)$ \\
\hline 80 & 84.7 & 3.1 \\
\hline 0.0 & -4.1 & 3.0 \\
\hline
\end{tabular}

In case of the low-temperature Nitinol, particular care was devoted to the integration of the Nitinol wire into the tag and to have the conducting glue getting solid. The fabrication of the switch was hence performed inside the thermostatic chamber itself.

In both cases the measurements have been repeated five times to perform averaging. Examples of collected data in both the experiments are shown in Fig. 11 and Fig. 12 and the estimated switch-off temperatures can be found in Table II.

The standard deviation is the same $\left(3^{\circ} \mathrm{C}\right)$ in both the configurations even if it is percentually more significant in the low-temperature case. This is probably due to the more difficult integration of the nitinol wire at temperature below zero degrees. The conducting glue adhesion reliability proved to be poor due to the presence of water/ice condensation between the nitinol and copper trace which makes it difficult to replicate the same device conditions in successive tests.

\section{CONCLUSIONS}

The Nitinol compound demonstrates to be suitable for integration into UHF tags for the control of both cold and hot goods 
and its response to temperature variation seems to be stable and repeatable, especially at high temperatures.

The experiments however highlight the importance of having a stable fastening method for the Nitinol wire to the antenna and further research is required to improve the stability of the device, particularly below $0^{\circ} \mathrm{C}$. Moreover the self-sensing tag should be provided with a lock mechanism to keep the Nitinol wire in the curved shape before use even at temperatures exceeding the austenite transition. This is also important in case the tags are placed over the items before they freeze.

Finally, the described design methodology for two-chip tags is more general than the considered temperature application, and it may be extended to the integration of other families of passive sensors, provided that their impedance model is known and that the fitness function is properly defined according to the required data encoding.

\section{ACKNOWLEDGMENT}

The authors wish to thank F. Nanni and C. Del Gaudio for inspiration and support on high-temperature measurements, SAES-GETTER for providing Nitinol wires and hosting the low-temperature experiments and finally but not least, G. Manzi of NXP for technical support with microchips and readers.

\section{REFERENCES}

[1] D. M. Dobkin, The RF in RFID: Passive UHF RFID in Practice. Amsterdam: Elsevier, 2007.

[2] Y. Li, Z. Rongwei, D. Staiculescu, C. P. Wong, and M. M. Tentzeris, "A novel conformal RFID-enabled module utilizing inkjet-printed antennas and carbon nanotubes for gas-detection applications," IEEE Antennas Wireless Propag. Lett., vol. 8, pp. 653-656, 2009.

[3] J. Sidn, X. Zeng, T. Unander, and H.-E. Nilsson, "Remote moisture sensing utilizing ordinary RFID tags," in Proc. IEEE Sensors, Atlanta, GA, Oct. 2007, pp. 308-311.

[4] J. Virtanen, L. Ukkonen, and L. Sydnheimo, "Ink jet printed RFID humidity sensor," presented at the RFIDay 2010, Tampere, Finland.

[5] A. Vaz, A. Ubarretxena, I. Zalbide, D. Pardo, H. Solar, A. Garca-Alonso, and R. Berenguer, "Full passive UHF tag with a temperature sensor suitable for human body temperature monitoring," IEEE Trans. Circuits Syst.-II_Expr. Briefs, vol. 57, no. 2, pp. 95-99, Feb. 2010

[6] R. Bhattacharyya, C. Floerkemeier, and S. Sarma, "RFID tag antenna based temperature sensing," in Proc. IEEE Int. Conf. on RFID, 2010, pp. $8-15$.

[7] M. C. O'Connor, "Coldchain project reveals temperature inconsistencies," RFID J. Dec. 2006 [Online]. Available: http://www.rfidjournal. com/article/view/2860/1

[8] A. Dada and F. Thiesse, "Sensor applications in the supply chain: The example of quality-based issuing of perishables," Lecture Notes Comput. Sci., vol. 4952, pp. 140-154, 2008.

[9] R. Kuchta and R. Vrba, "Wireless and wired temperature data system," in Proc. Int. Conf. on Systems ICONS, 2007, p. 49.

[10] S. Kim, J. H. Cho, H.-S. Kim, H. Kim, H. B. Kang, and S. K. Hong, “An EPC Gen 2 compatible passive/semi-active UHF RFID transponder with embedded FeRAM and temperature sensor," in Proc. IEEE Asian Solid-State Circuits Conf., Jeju, Korea, 2007, pp. 4-6.

[11] J. Gao, J. Sidn, and H. E. Nilsson, "Printed temperature sensors for passive RFID tags," presented at the Progress In Electromagnetics Research Symp., Xi' an, China, 2010.

[12] M. Philipose, J. R. Smith, B. Jiang, A. Mamishev, S. Roy, and K. Sundara-Rajan, "Battery-free wireless identification and sensing," IEEE Pervasive Comput., vol. 4, no. 1, pp. 37-45, 2005.

[13] H. Funakubo, Shape Memory Alloys. New York: Gordon and Breach Publishers, 1987.
[14] E. Hornbogen, "Review thermo-mechanical fatigue of shape memory alloys," J. Mater. Sci., vol. 39, no. 2, pp. 385-399, 2004.

[15] AA.VV, Niti Smart Sheet-Properties [Online]. Available: http:// web.archive.org/web/20030418012213/http://www.sma-inc.com/NiTiProperties.html

[16] E. B. Rosa, "The self and mutual inductances of linear conductors," Bulletin Bureau of Stand., vol. 4, no. 2, p. 301, 1908.

[17] G. Marrocco, L. Mattioni, and C. Calabrese, "Multi-port sensor RFIDs for wireless passive sensing-Basic theory and early simulations," IEEE Trans. Antennas Propag., vol. 56, no. 8, pp. 2691-2702, Aug. 2008.

[18] J. R. Mautz and R. Harrington, "Modal analysis of loaded N-port scatterers," IEEE Trans. Antennas Propag., vol. 21, no. 2, pp. 188-199, March 1973

[19] G. Marrocco, "Electromagnetic theory of RFID grids," IEEE Trans. Antennas Propag., submitted for publication.

[20] C. A. Desoer, "The maximum power transfer theorem for n-ports," IEEE Trans. Circuit Theory, vol. 20, no. 3, pp. 328-330, May 1973.

[21] B. Yang and Q. Feng, "A folded dipole antenna for RFID tag," in Proc. Int. Conf. on Microwave and Millimeter Wave Technology, 2008.

[22] G. Marrocco and F. Bardati, "BEST: A finite-difference solver for time electromagnetics," Simulation Practice Theory, no. 7, pp. 279-293, 1999.

[23] G. Marrocco and F. Amato, "Self-sensing passive RFID: From theory to tag design and experimentation," in Proc. 39th Eur. Microwave Conf., Rome, 2009, pp. 1-4.

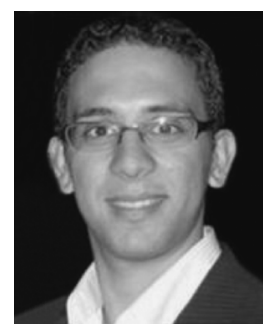

Stefano Caizzone received the M.Sc. degree in telecommunications engineering from the University of Rome "Tor Vergata," in 2009, where he is working part-time toward the Ph.D. degree.

His main research interests concern small antennas for RFIDs and navigation, antenna arrays and grids with enhanced sensing capabilities. He is now with the Antenna Group, Institute of Communications and Navigation, German Space Agency (DLR), where he is responsible for the development of innovative miniaturized antennas.

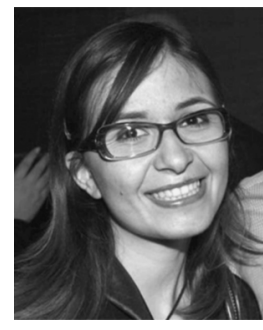

Cecilia Occhiuzzi received the M.Sc. degree in medical engineering from the University of Rome "Tor Vergata," where she is currently working toward the Ph.D. degree.

In 2008, she was at the School of Engineering, University of Warwick, U.K., as a Postgraduate Student, working on design and implementation of wireless SAW sensors. In 2010, she was a Visiting Researcher at the Georgia Institute of Technology, Atlanta. Her research was mainly focused on the design of passive RFID sensors for structural health monitoring and gas detection by means of CNT-based tags.

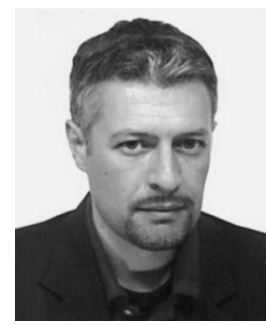

Gaetano Marrocco was born in Teramo, Italy, on August 29, 1969, He received the Laurea degree in electronic engineering (laurea cum laude and academic honour) and the Ph.D. degree in applied electromagnetics from the University of L'Aquila, Italy, in 1994 and 1998, respectively.

Since 1997, he has been a Researcher at the University of Rome "TorVergata," Rome, Italy, where he currently teaches antenna design and medical radiosystems, manages the Antenna Lab and is Advisor in the Geo-Information Ph.D. program. In October 2010, he achieved the degree of Associate Professor of Electromagnetics. In summer 1994, he was at the University of Illinois at Urbana-Champaign as a 
Postgraduate Student. In autumn 1999, he was a Visiting Researcher at the Imperial College in London, U.K. In 2008, he joined the Ph.D. program of the University of Grenoble (FR). His research is mainly directed to the modeling and design of broad band and ultrawideband (UWB) antennas and arrays as well as of sensor-oriented miniaturized antennas for biomedicine, aeronautics and radiofrequency identification (RFID). He has been involved in several Space, Avionic, Naval and Vehicular programs of the European Space Agency, NATO, Italian Space Agency, and the Italian Navy about the analysis and the design of non conventional antennas and systems. He holds eight patents on broadband naval antennas and structural arrays, and on sensor RFID systems.
Prof. Marrocco currently, he serves as an Associate Editor of the IEEE ANTENNAS AND WIRELESS PROPAGATION LETTERS and is a Reviewer for the iEEE TRANSACtion on ANTENNAS and PROPAgation, IEEE ProceEdings, IEEE MicROWAVE THEORY AND TECHNIQUES, and is a member of Technical Program Committee of several International Conferences. In 2008, he was the General Chairman of the first Italian multidisciplinary scientific workshop on RFID: RFIDays-2008: Emerging Technology for Radiofrequency Identification. He was the Co-Chair of the RFIDays-2010 International Workshop in Finland and Chairman of the Local Committee of the V European Conference on Antennas and Propagation. 